\title{
Trans-oral robotic surgery for obstructive sleep apnea - selection of patients
}

\author{
Mario Milkov \\ Department of Dental Material Science and Propaedeutic of Prosthetic Dental Medicine, \\ Faculty of Dental medicine, Medical University - Varna
}

\begin{abstract}
:
The incidence of obstructive sleep apnea-hypopnea syndrome (OSAHS) is about 7\% in middle-aged adults. OSAHS remains underdiagnosed worldwide, delaying treatment with subsequent onset of complications, mainly cardiac and neurological. The surgical management of OSAHS is appealing to both patients and providers as a single intervention that could limit health care costs and avoid the use of continuous positive airway pressure. Technological advancement has allowed tongue base reduction causing airway obstruction to be addressed using TORS safely and with ease. The purpose of this study is to discuss appropriate patient selection when considering trans-oral robotic surgery (TORS) as surgical treatment of OSAHS.

Keywords: obstructive sleep apnea-hypopnea syndrome, trans-oral robotic surgery, selection of patients, criteria
\end{abstract}

\section{Introduction}

The incidence of obstructive sleep apnea-hypopnea syndrome (OSAHS) is about $7 \%$ in middle-aged adults $[24,34]$. Young et al. found that the prevalence was 3-28\% in the general population [42]. In a US study published in 2012, the prevalence of moderate to severe OSAHS was $10 \%$ for men aged $30-49$ years and $17 \%$ for women aged $50-70$ years, with $3 \%$ and $9 \%$ for women, respectively [28]. The authors found that the prevalence increased strongly by $14-55 \%$ over a 20 -year period, depending on OSAHS age, gender, and severity [28].

The first data on the disease date back to the 18th century, but it was not until the 1970s that OSAHS became a center of interest [15]. OSAHS remains underdiagnosed worldwide, delaying treatment with subsequent onset of complications, mainly cardiac and neurological [13, 33].

The surgical management of OSAHS is appealing to both patients and providers as a single intervention that could limit health care costs and avoid the use of continuous positive airway pressure (CPAP). While CPAP is an extremely effective in the treatment of OSAHS, its tolerability, adherence to its use and the social stigma make it difficult for many patients. Despite multiple available procedures to surgically treat OSAHS, current treatments continue to rely on careful patient selection and appropriate application of surgical interventions.

The purpose of this study is to discuss appropriate patient selection when considering trans-oral robotic surgery (TORS) as surgical treatment of OSAHS. 


\section{Comorbidity}

The preoperative and perioperative management of patients with OSAHS is challenging, as often these patients will have coexisting illnesses such as systemic hypertension, systolic and diastolic myocardial dysfunction, insulin resistance, pulmonary hypertension, stroke, coronary artery disease, and cardiac arrhythmias.

The best studied cardiovascular comorbidity in OSAHS is systemic hypertension [27]. Respiratory events during sleep are associated with hypertensive peaks occurring at the end of apneas and hypopneas, increased mean nocturnal blood pressure, and increased variability of blood pressure [22]. A dose-response relationship has been shown between OSAHS severity and blood pressure [44]. Several studies have addressed the question of OSAHS and cardiovascular morbidity and mortality. The results of observational studies confirmed the association of untreated OSA with overall and cardiovascular mortality [12].

Arrhythmias are frequent in OSAHS patients, especially atrial fibrillation (AF). A permissive role of OSAHS towards the arrhythmogenic mechanism of $\mathrm{AF}$ is suggested by the higher risk of recurrence of AF in patients with OSAHS [5, 8].

Several studies reported an increased risk of stroke in snorers [19] and OSA patients [30]. Several wellconducted studies have demonstrated the association, with an adjusted relative risk of $2.86(95 \% \mathrm{CI}$, 1.1-7.4) for stroke in males with apnea/hypopnea index $>19 \mathrm{~h}$ [30], and high prevalence $(54 \%)$ of OSAHS found in patients presenting with a first episode of acute coronary syndrome [32].

The relationship between OSAHS and metabolism is highly complex. On one hand, OSAHS is often associated with obesity, which by itself is characterized by disturbed energy metabolism and adipose tissue inflammation [31]. On the other hand, nocturnal intermittent hypoxia has been shown to affect glucose metabolism, and OSAHS could independently contribute to the pathogenesis of metabolic disorders [2]. Meslier et al. showed that the prevalence of diabetes and of glucose intolerance was significantly elevated in OSAHS patients (at respectively $30.1 \%$ and $20 \%$ ), with a significant correlation between insulin resistance and apnea/ hypopnea index [23].

Several studies have described perioperative complications associated with OSAHS which include higher reintubation rates, hypercapnia, oxygen desaturations, cardiac arrhythmias, myocardial injury, delirium, unplanned ICU transfers, and longer hospitalization stays [1]. Moreover, the administration of anesthesia exacerbates the upper airway anatomic alterations that lead to pharyngeal collapse in patients with OSAHS. Therefore, appropriate measures should be taken to identify and treat patients with OSAHS and comorbidities to reduce their perioperative risk. Patients with a lower American Society of Anesthesiologists (ASA) score, which measures a patient's systemic fitness before surgery, less than three have been found to have greater success with TORS for OSAHS [17].

Preoperative management should begin with a detailed history of physical exam with emphasis on airway examination as mask ventilation and tracheal intubation are often more difficult in patients with OSAHS [35]. Comorbidities such as hypertension, diabetes mellitus, and congestive heart failure should be assessed for adequacy of control. Any patient with uncontrolled hypertension, hyperglycemia, or decompensated heart failure should be referred to his or her primary care physician for medical optimization prior to surgery [29].

\section{Polysomnography}

The gold standard for the diagnosis of OSAHS is polysomnography (PSG). The test is monitored and conducted at specialized laboratories, keeping record of multiple physiologic characteristics: electroenchephalogram, electrooculogram, chinelectromyogram, air flow (through nasal pressure or thermostat), abdominal and thoracic wall movement, body position, electrocardiography and oxygen saturation. Additionally, polysomnography exams are usually followed by continuous positive airway pressure (CPAP) titration test.

A variety of sleep studies are available, including Type 1 attended PSG in which multiple measurements are collected including EEG, ECG, EMG, oxygen saturation, airflow monitoring, chest movement, blood pressure, and positioning during sleep. Type 2 studies are unattended and measure at least seven of these parameters. Type 3 and 4 studies are unattended and measure a minimum of 4 and 3 channels, respectively. There are portable (Type 2-A) monitoring systems available and these are used in countries such as Italy, where formal PSG is reserved for selected patients (5\%). The sleep study report includes the apnea/hypopnea index 
(AHI), or the number of apneas and hypopneas that occur per hour. Other factors include sleep latency, duration, efficiency, and snoring. The respiratory disturbance index (RDI) includes all events (not only apneas and hypopneas) that occur per hour. Taken together, the PSG defines the severity of OSAHS and can help guide the clinician to identify patients who may be successful with surgical interventions [16].

Mild OSAHS is defined as 5-14 AHI events per hour, moderate OSAHS with 15-29 AHI events per hour, and severe OSAHS with $>30$ AHI events per hour. Patients with moderate to severe OS AHS $($ AHI > 20) are potential candidates for TORS; patients with mild to moderate OS AHS (AHI 5-15 and 16-30) may be amenable to other treat- ments including oral appliance or tonsillectomy and uvulopalatopharyngoplasty/lateral expansion pharyngoplasty with or without thyro-hyoidopexy $[16,26]$.

In a review of the patients who underwent TORS \pm multilevel surgery for OSAHS, it was found that patients with severe OSAHS had more improvement, success, and cure 3 months after surgery than their moderate counterparts (Fig 1) [17]. Improvement was defi ned as any decrease in preoperative apnea hypopnea index (AHI). Success was defi ned as both AHI $<20$ and decrease by $50 \%$. Cure was defi ned as $\mathrm{AHI}<5$. In general, patients with severe OSAHS (defi ned as AHI $\geq$ 30) achieved greater improvement, success, and cure rates than those with moderate OSAHS (AHI

\section{5-29) [17]}

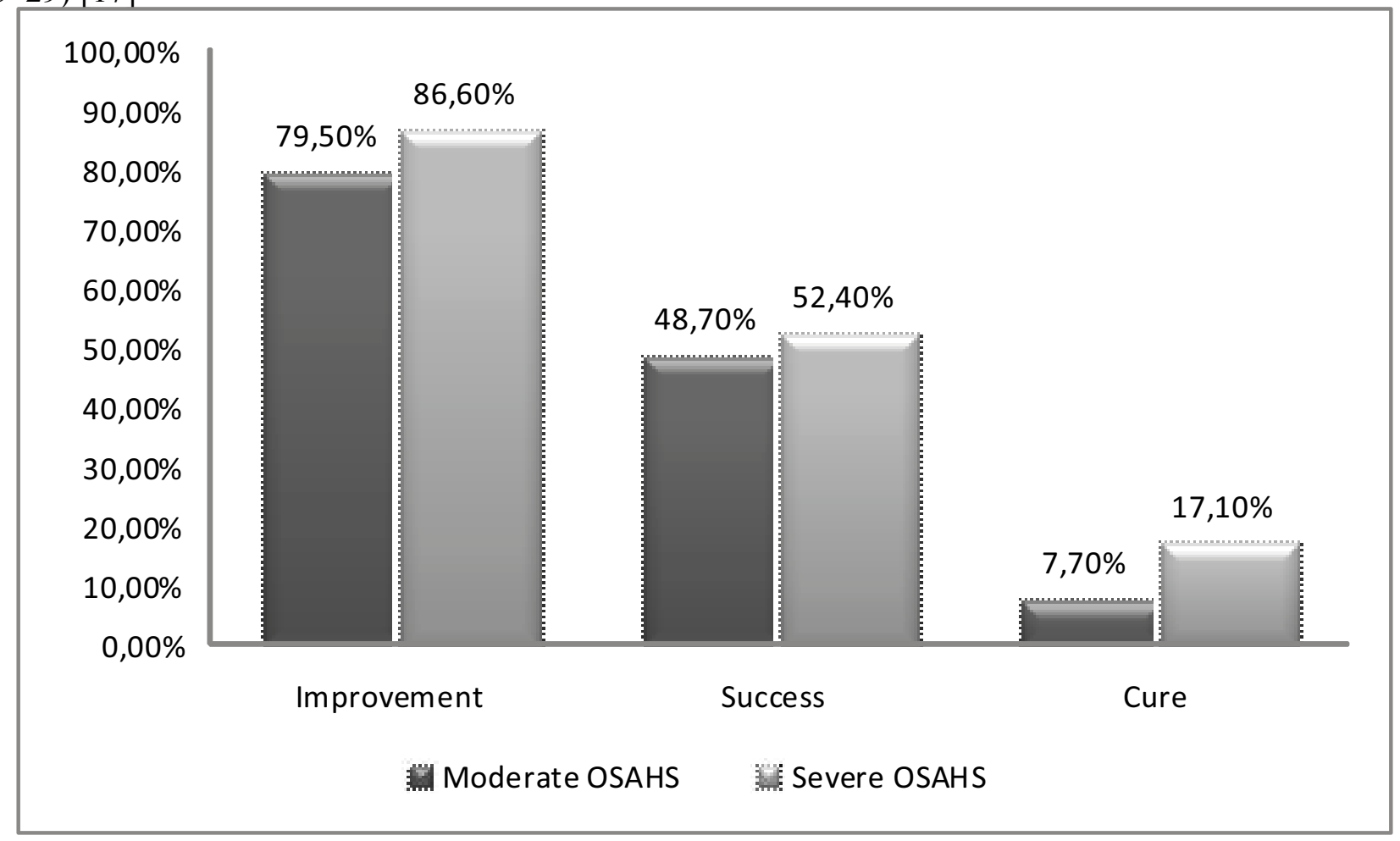

Fig. 1. A retrospective review of the patients who underwent trans-oral robotic surgery (TORS) \pm multilevel surgery for obstructive sleep apnea hypopnea syndrome (OSAHS)

\section{Subjective Measurements}

Subjective measurements of sleepiness and snoring have been developed and are commonly used as a measure of treatment success. The Epworth sleepiness scale is a 24-point scale measuring subjective sleepiness in eight different scenarios. The Thornton snoring scale is a similar scale in which the patient evaluates the effect of disruptive snoring on his or her relationship in different scenarios. Both should be measured before and after surgery. More comprehensive measures of quality of life include the functional outcome of sleepiness questionnaire (FOSQ) and the shortened FOSQ-10. These questionnaires have been validated and are easy to administer.

A careful assessment of preoperative swallowing function should be performed. The Dysphagia handicap index or the MD Anderson Dysphagia Index will help to avoid the mistake of performing 
tongue base surgery on patients with underlying pathology that affects swallowing function-most commonly due to advanced age. Documentation of preoperative and postoperative swallowing is important particularly if dysphagia is due to obstructing lingual tonsils.

Tabl. 1. Epworth Sleepiness Scale [18]

\begin{tabular}{|c|c|c|c|}
\hline Situation & $\begin{array}{l}\text { Chance of } \\
\text { dozing }\end{array}$ & Points & Results \\
\hline Sitting and reading & & $\begin{array}{l}0- \\
\text { No chance of dozing }\end{array}$ & $\begin{array}{l}0-10 \\
\text { Normal }\end{array}$ \\
\hline Watching TV & & $\begin{array}{l}1- \\
\text { Slight chance of dozing }\end{array}$ & $\begin{array}{l}\text { 11-12 } \\
\text { Borderline }\end{array}$ \\
\hline $\begin{array}{l}\text { Sitting inactive in a public place (e.g. a theater or a } \\
\text { meeting) }\end{array}$ & & $\begin{array}{l}2- \\
\text { Moderate chance of dozing }\end{array}$ & $\begin{array}{l}>12 \\
\text { Abnormal }\end{array}$ \\
\hline \multicolumn{4}{|l|}{ As a passenger in a car for an hour without a break } \\
\hline \multicolumn{4}{|l|}{$\begin{array}{l}\text { Lying down to rest in the afternoon when circumstances } \\
\text { permit }\end{array}$} \\
\hline \multicolumn{4}{|l|}{ Sitting and talking to someone } \\
\hline \multicolumn{4}{|l|}{ Sitting quietly after lunch without alcohol } \\
\hline In a car, while stopped for a few minutes in traffic & & & \\
\hline
\end{tabular}

\section{Physical Exam Findings (Table 2)}

Tabl. 2. Physical exam findings that should be documented in the preoperative assessment of candidacy for surgery for obstructive sleep apnea hypopnea syndrome

\begin{tabular}{l}
\hline Important physical exam fi ndings \\
\hline Nasal obstruction \\
Inter-incisal distance and dentition \\
Angle class \\
Friedman palate position \\
Tonsil size \\
Modified Cormack-Lehane view \\
Lingual tonsil size \\
\hline
\end{tabular}

\section{Nasal Examination}

In-office physical examination of the upper airway is essential to successful TORS. Severe nasal obstruction due to septal deformity may preclude transnasal intubation. Any nasal deformity including deviated septum, inferior turbinate hypertrophy, and adenoid hypertrophy should be addressed at the time of drug induced sleep endoscopy (DISE). Radiofrequency ablation of the inferior turbinates can be done as an in-office procedure or added to DISE without the need for general anesthesia [16].

\section{Examination for Access}

Not all patients are suitable candidates for TORS. Appropriate exposure of the target region is a key factor. The target region will invariably be difficult to remove if its boundaries are not visible. Suboptimal access has major implications for poor outcome, such as a higher risk of damage to healthy adjacent structures, or abandoning the procedure. Several clinical studies have highlighted the importance of careful patient selection [7,9]. In a recent study to optimize laryngeal and hypopharyngeal exposure, De Virgilio et al. reported a 100\% success rate in their ability to perform TORS when an examination under anesthetic (EUA) was carried out beforehand. Despite this meticulous approach, failed TORS due to inadequate target exposure has between reported to occur in $7-26 \%$ of cases $[37,41]$. Therefore, predictive metrics are a useful adjunct in the preoperative clinical setting to confirm adequate access to the target region and reduce the percentage of unanticipated TORS failures.

In a recent cadaver study, three TORS surgeons independently evaluated feasibility using 51 Caucasian cadavers [3]. In order to improve tissue pliability, a phenol-glycerol technique was used to embalm the cadavers. Trans-oral visualization was performed with two retractors commonly used in TORS. Seven anthropometric parameters and the degree of mouth opening were recorded. The results suggest that biometric measures of the extent of mouth-opening, neck circumference, hyoid- mental length, and mandibular body height may provide an important tool in the decisionmaking process when assessing patient suitability for TORS. The type of retractor used in this study appeared to play an important role in the variability of target exposure. Clinical validation in a large patient cohort is necessary because this study does not account for important factors that affect 
decision-making in clinical practice such as DISE findings, patient factors (e.g., age and performance status) and surgical experience. When making a clinical decision to proceed with TORS, all of these factors need to be collectively considered to determine patient suitability.

Preoperative cephalometric measurements obtained from preoperative imaging are also useful [21]. Inter-incisor distance and dentition should be carefully assessed. Most patients have an interincisal distance greater than $4 \mathrm{~cm}$; less than $2.5 \mathrm{~cm}$ generally precludes successful TORS.

For those patients affected by malignant neoplasms, TORS feasibility is qualitatively assessed by performing an examination under anesthesia (EUA) using a mouth gag (Davis-Mayer ${ }^{\circledR}$ Karl Storz, Germany or Boyle-Davis ${ }^{\circledR}$ Surgical Holdings, Essex, UK) or a $\mathrm{FK}-\mathrm{WO}^{\circledR}$ retractor (Olympus), with the patient in a standardized position (supine with neck extended and head flexed). The EUA includes visualization of the tonsils, tongue base, and supraglottis. Patients with trismus or retrognathia (Angle class II) make the placement of the robotic arms and camera extremely challenging. In patients affected by OSA, if there is marginal accessibility as deter-mined by clinical and cephalometric evaluation, it is suggested that the mouth gag position will be evaluated at the time of DISE to confirm candidacy for TORS.

\section{Friedman Staging System}

In an effort to predict success in patients undergoing sleep surgery, Friedman developed a staging system based on tongue position and tonsil size in 2002 [11]; this system has become the standard by which sleep surgeons counsel their patients. The Friedman Staging system categorizes patients based on visualization of the tonsillar pillars, uvula, soft, and hard palate with the oropharynx in a natural position [10]. Tongue position (Grade 1-4) is based on a modification of the Mallampati grade used by anesthesiologists in assessing tongue position; the modification classifies tongue position in the resting position (not protruded) with the mouth wide open. Tonsil size is graded 1-4. Along with BMI, this staging system was developed with the goal of predicting surgical success in patients with OSAHS. In a recent study of patients who undergo TORS \pm multilevel procedures for OSAHS, after stratifying by preoperative Friedman stage, success was seen in $75 \%$ of stage I, $70 \%$ of stage II, $66 \%$ of stage III, and $10 \%$ of stage IV patients. When stratifying by preoperative BMI, success was seen in $75 \%$ of stage II and $72 \%$ of stage III patients with preoperative BMI $<30$, compared to $58 \%$ of stage II and $56 \%$ of stage III patients with preoperative $\mathrm{BMI}>30$ [36].

\section{Modified Cormack-Lehane View}

Modified Cormack-Lehane view can also help assess patient candidacy for TORS. The CormackLehane classification was developed as a tool for anesthesiologists as they described the view of the larynx during direct laryngoscopy. A modification has been created using a flexible nasopharygoscope which gives the observer a physiologic view of the anatomy during the awake state [16].

\section{Lingual Tonsil Examination}

Grading the size of the lingual tonsil is important in assessing candidacy for surgery. To grade lingual tonsils, the tongue should be in the resting position, with the mouth closed as occurs in sleep. The patient should be in the supine position. Comparison of awake and sedated lingual tonsil size can be done during DISE; however the final grade should be assessed with the patient awake and seated. Lingual tonsils are given a grade of $0-4$ (Grade 0 lingual tonsils are not visible or absent, Grade 1 scattered, Grade 2 covers BOT but minimal verticle height, Grade 3 fills vallecula but does not displace epiglottis, Grade 4 fills vallecula and extends above tip of the epiglottis). Grade 3 or 4 upright or supine are considered good surgical candidates. The best candidates for TORS have discrete areas of lingual tonsil hypertrophy low in the vallecula, and/or secondary collapse of the epiglottis due to direct contact with enlarged lingual tonsil. Patients with a large, muscular tongue (Friedman stage 3 or 4 ) and minimal lingual tonsil tissue are typically poor candidates for TORS. Many of the latter patients also have a BMI > 30; a large tongue is often due to fatty infiltration in obese patients [25].

\section{Body Mass Index}

Obesity was the main factor behind this increase in the prevalence of OSAHS. Central obesity, which generates fat distribution particularly at the abdominal level, upper body and neck, is the one most associated with OSAHS [38, 43]. Despite ideal anatomic criteria for TORS, cure of OSAHS in obese patients remains elusive, and surgeons 
should not raise unrealistic expectations for success in these patients. Vicini et al. were the first authors to show that TORS can show an efficacy of $75 \%$ at 6 months in patients with a body mass index (BMI) under 30, but as BMI increased, the success of TORS fell to 55\% [39]. In a multivariate analysis of over 120 patients who underwent TORS for OSAHS, BMI was shown to predict success (defined as postoperative AHI $<20$ and decrease in AHI by $50 \%$ ). Patients with a BMI under 30 can expect the best results ( $86 \%$ of patients show improvement, $69 \%$ have success, $15 \%$ are cured). In fact, operative success was inversely correlated with preoperative BMI as shown in Fig. 2 [17]. Success in surgery for obstructive sleep apnea hypopnea syndrome (OSAHS) is achieved if the patient obtains a postoperative apnea hypopnea index $(\mathrm{AHI})<20$ and a decrease from preoperative AHI by $50 \%$. In a study of the patients who underwent trans-oral robotic surgery for OSAHS, success was inversely correlated with preoperative body mass index (BMI) [17].

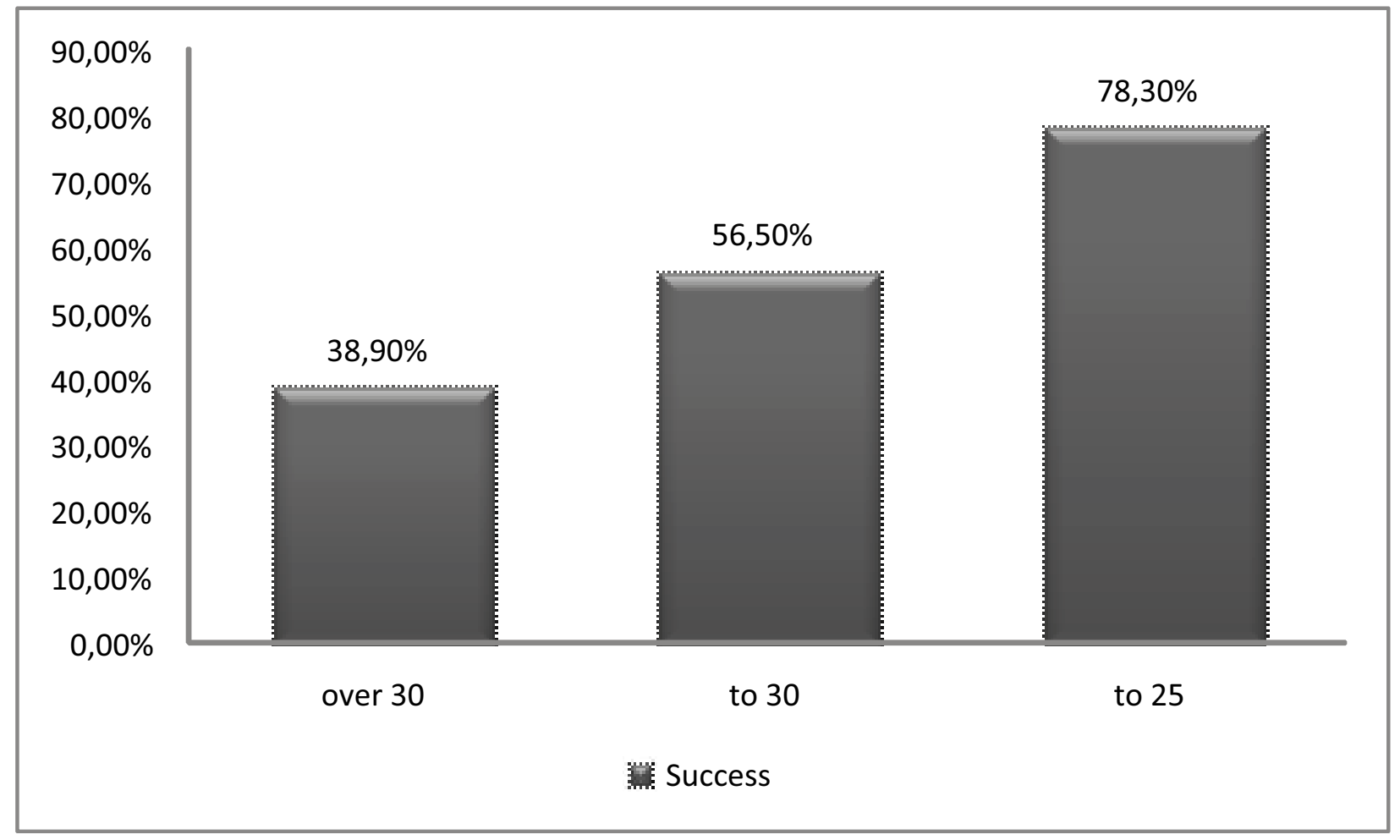

Fig. 2. BMI and success from trans-oral robotic surgery for OSAHS

It is advisable to encourage obese patients to enter a medical weight loss program while using CPAP prior to surgery. Bariatric surgery may also be an option for these patients. Bariatric surgery is a very effective tool for the treatment of OSAHS, with a success rate of $70 \%$ in a meta-analysis of 12 studies of patients with moderate to severe OSAHS [14]. PSG should be repeated after targeted weight loss has been achieved.

A reduction in BMI following upper airway surgery can confound the surgical outcome. This may be true for patients undergoing TORS, where reduced oral intake from dysphagia and odynophagia may cause a weight reduction.

Studies that looked at changes before and after TORS did not reveal statistical changes in BMI to account for the reduction in AHI. Hoff et al. [17] found that patients with a lower preoperative BMI had a higher chance of surgical success: the proportion of patients who had surgical success with BMIs of $<30$ and $<$ 25 were 56.5 and $78.3 \%$, respectively. Lin et al. [20] found that patients with a BMI $<30$ respond better than those with a BMI $>40$. The surgical response rate of patients with a $\mathrm{BMI}<30$ is $88.2 \%$ and those with a BMI $>40$ is $16.7 \%$.

\section{Drug Induced Sedation Endoscopy (DISE)}

In brief, it was first described in 1991 by Croft and Pringle and allows the treating physician to observe areas of physical obstruction that occur during induced sleep [6]. The examiner evaluates the airway in a very methodical fashion with the 
patient both awake and sedated [40]. In 2014, The European Sleep Society presented a position paper and had consensus on many fundamental aspects of DISE, but were unable to agree upon either a unified classification system or a scoring system for DISE. There are two widely used grading systems (VOTE and NOHL). The sites examined include the nasopharynx (velum), oropharynx, hypopharynx (tongue), and larynx or epiglottis (both supraglottic and glottic). For each site, the degree (grade) of obstruction $(<25 \%$ grade I, $25-50 \%$ grade II, $50-75 \%$ grade III. and $>75 \%$ grade IV), pattern (concentric, ap $=$ anterior posterior, $1=$ lateral), and mechanics (vibration, collapse) are recorded. With a better understanding of the multilevel obstruction that characterizes obstructive episodes, surgeons can identify areas that may be amenable to surgical treatment. DISE often reveals areas of obstruction that were not anticipated during office examination and may significantly change the surgical plan [4]. In addition, the patients appreciate the opportunity to review the findings during a separate office consultation, where review of the video and surgical plan is outlined.

\section{Patient Profile for TORS}

The challenge that has always faced sleep surgeons has been the ability to identify those patients with
OSAHS who will benefit from surgery and those who should be counseled to look at other options. Based on the authors' experience as well as a review of the literature, the ideal preoperative criteria for TORS for OSAHS are listed in Table 3.

Tabl. 3. Patient Profile for TORS

\begin{tabular}{l}
\hline Polysomnography showing moderate to severe \\
OSAHS (AHI > 15) \\
\hline Age $>18$ years \\
BMI $<30$ \\
Failed trial of CPAP \\
Failed surgery \\
Intraoral accessibility \\
Identifiable target areas seen on DISE \\
\hline
\end{tabular}

\section{Conclusion}

Technological advancement has allowed tongue base reduction causing airway obstruction to be addressed using TORS safely and with ease. It is merely a tool to allow resection of the tongue base under direct vision and allows the surgeon to work around corners in the oral cavity and hypopharynx. Safe resection of an obstructing tongue base achieves improved AHI, ESS, and LSAT, with acceptable morbidity. An appropriate patient selection is paramount in yielding desirable results.

\section{References}

1. Adesanya AO, et al. Perioperative management of obstructive sleep apnea. Chest. 2010; 138(6): 1489-98.

2. Anothaisintawee T, Reutrakul S, Van Cauter E, Thakkinstian A. Sleep disturbances compared to traditional risk factors for diabetes development: Systematic review and meta-analysis. Sleep Med Rev. 2016 Dec;30:11-24. https://doi.org/10.1016/j.smrv.2015.10.002

3. Arora A, Kotecha J, Acharya A, Garas G, Darzi A, Davies DC, Tolley N. Determination of biometric measures to evaluate patient suitability for transoral robotic surgery. Head Neck. 2015;37(9):1254-60.

4. Campanini A, et al. Awake versus sleep endoscopy: personal experience in 250 OSAHS patients. Acta Otorhinolaryngol Ital. 2010; 30(2): 73-7.

5. Congrete S, Bintvihok M, Thongprayoon C, Bathini T, Boonpheng B, Sharma K, Chokesuwattanaskul R, Srivali N, Tanawuttiwat T, Cheungpasitporn W. Effect of obstructive sleep apnea and its treatment of atrial fibrillation recurrence after radiofrequency catheter ablation: A meta-analysis. J Evid Based Med. 2018;11:145-51. https://doi.org/10.1111/jebm.12313

6. Croft CB, Pringle M. Sleep nasendoscopy: a technique of assessment in snoring and obstructive sleep apnoea. Clin Otolaryngol Allied Sci. 1991; 16(5): 504-9.

7. De Virgilio A, Park YM, Kim WS, Baek SJ, Kim SH. How to optimize laryngeal and hypopharyngeal exposure in transoral robotic surgery. Auris Nasus Larynx. 2013; 40: 312-9.

8. Deng F, Raza A, Guo J. Treating obstructive sleep apnea with continuous positive airway pressure reduces risk of recurrent atrial fibrillation after catheter ablation: a meta-analysis. Sleep Med. 2018 Jun;46:5-11. https://doi.org/10.1016/j.sleep.2018.02.013

9. Dowthwaite S, Nichols AC, Yoo J, et al. Transoral robotic total laryngectomy: report of 3 cases. Head Neck. 2013 ; 35 (11): E338-42.

10. Friedman M, et al. Clinical predictors of obstructive sleep apnea. Laryngoscope.1999; 109(12): $1901-7$.

11. Friedman M, Ibrahim H, Bass L. Clinical staging for sleep-disordered breathing. Otolaryngol Head Neck Surg. 2002 ; $127(1)$ : 13-21.

12. Fu Y, Xia Y, Yi H, Xu H, Guan J, Yin S. Meta-analysis of all-cause and cardiovascular mortality in obstructive sleep apnea with or without continuous positive airway pressure treatment. Sleep Breath. 2017;21:181-9. https://doi.org/10.1007/s11325-016-1393-1

13. Gibson GJ. Obstructive sleep apnoea syndrome: underestimated and under-treated. Br Med Bull 2004; 72(Jan (1)): 49-64. 
14. Greenburg DL, Lettieri CJ, Eliasson AH. Effects of surgical weight loss on measures of obstructive sleep apnea: a meta-analysis. Am J Med. 2009; 122(6): 535-42.

15. Guilleminault C, Tilkian A, Dement WC. The sleep apnea syndromes. Annu Rev Med 1976; 27(1): 465-84.

16. Hoff PT, Spector ME. In: Sood M, Leichtle SW, editors. Essentials of robotic surgery. Ann Arbor, MI: Spry Publishing; 2013.

17. Hoff PT, Glazer TA, Spector ME. Body mass index predicts success in patients undergoing transoral robotic surgery for obstructive sleep apnea. ORL J Otorhinolaryngol Relat Spec. 2014; 76(5): 266-72.

18. Johns M. A new method for measuring daytime sleepiness. Sleep 1991; 14: 540-545.

19. Li M, Li K, Zhang X-W, Hou W-S, Tang Z-Y. Habitual snoring and risk of stroke: a meta-analysis of prospective studies. Int J Cardiol. 2015; 185: 46-9. https://doi.org/10.1016/j.ijcard.2015.03.112

20. Lin HS, Rowley JA, Folbe AJ, Yoo GH, Badr MS, Chen W: Transoral robotic surgery for treatment of obstructive sleep apnea: factors predicting surgical response. Laryngoscope 2015; 125: 1013-1020.

21. Luginbuhl A, Baker A, Curry J, Drejet S, Miller M, Cognetti D. Preoperative cephalometric analysis to predict transoral robotic surgery exposure. J Robot Surg. 2014; 8: 313-7.

22. Marrone O, Bonsignore MR. Blood-pressure variability in patients with obstructive sleep apnea: current perspectives. Nat Sci Sleep. 2018; 10: 229-42. https://doi.org/10.2147/NSS.S148543

23. Meslier N, Gagnadoux F, Giraud P, Person C, Ouksel H, Urban T, et al. Impaired glucose-insulin metabolism in males with obstructive sleep apnoea syndrome. Eur Respir J 2003; 22(Jul (1)): 156-60.

24. Meslier N, Vol S, Balkau B, Gagnadoux F, Cailleau M, Petrella A, et al. Prevalence des symptomes du syndrome d'apnees du sommeil. Etude dans une population franfaise d'age moyen. Rev Mal Respir 2007; 24(Mar (3, Part 1)): 305-13.

25. Nashi N, et al. Lingual fat at autopsy. Laryngoscope. 2007;117(8):1467-73.

26. Pang KP, Woodson BT. Expansion sphincter pharyngoplasty: a new technique for the treatment of obstructive sleep apnea. Otolaryngol Head Neck Surg. 2007;137(1):110-4

27. Parati G, Lombardi C, Hedner J, Bonsignore MR, Grote L, Tkacova R, Lŭvy P, Riha R, Bassetti C, Narkiewicz K, Mancia G, WT MN. EU COST Action B26 members. Recommendations for the management of patients with obstructive sleep apnoea and hypertension. Eur Respir J. 2013; 41: 523-38. https://doi.org/10.1183/09031936.00226711

28. Peppard PE, Young T, Barnet JH, Palta M, Hagen EW, Hla KM. Increased prevalence of sleep-disordered breathing in adults. Am J Epidemiol 2013; 177(May (9)):1006-14

29. Raveendran R, Chung F. Perioperative consideration of obstructive sleep apnea in ambulatory surgery. Anesthesiol Clin. 2014;32(2):321-8

30. Redline S, Yenokyan G, Gottlieb DJ, Shahar E, O'Connor GT, Resnick HE, et al. Obstructive sleep apnea-hypopnea and incident stroke. Am J Respir Crit Care Med 2010; 182(Jul (2)): 269-77

31. Schenk S, Saberi M, Olefsky JM. Insulin sensitivity: modulation by nutrients and inflammation. J. Clin. Invest. 2008; 118: 2992-3002. https:// doi.org/10.1172/JCI34260

32. Schiza SE, Simantirakis E, Bouloukaki I, Mermigkis C, Kallergis EM, Chrysos- tomakis S, et al. Sleep disordered breathing in patients with acute coronary syndromes. J Clin Sleep Med 2012; 8(Feb (1)) :21-6.

33. Shahar E, Whitney CW, Redline S, Lee ET, Newman AB, Javier Nieto F, et al. Sleep-disordered breathing and cardiovascular disease. Am J Respir Crit Care Med 2001; 163(Jan (1)): 19-25.

34. Simpson L, Hillman DR, Cooper MN, Ward KL, Hunter M, Cullen S, et al. High prevalence of undiagnosed obstructive sleep apnoea in the general population and methods for screening for representative controls. Sleep Breath 2012; 17(Nov(3)): 967-73.

35. Siyam MA, Benhamou D. Diffi cult endotracheal intubation in patients with sleep apnea syndrome. Anesth Analg. 2002; 95(4): 1098-102.

36. Spector ME, Glazer TA, Hoff PT. Addressing the retrolingual space in moderate to severe obstructive sleep apnea: outcomes stratifi ed by Friedman stage in patients undergoing TORS \pm multilevel surgery. ORL J Otorhinolaryngol Relat Spec. 2016; 78(1): 1-8.

37. Vergez S, Lallemant B, Ceruse P, et al. Initial multi-institutional experience with transoral robotic surgery. Otolaryngol Head Neck Surg. 2012; 147: 475-81.

38. Vgontzas A, Papanicolaou DA, Bixler EO, Hopper K, Lotsikas, A, Lin HM, Kales A, Chrousos GP. Sleep apnea and daytime sleepiness and fatigue: relation to visceral obesity, insulin resistance, and hypercytokinemia. J Clin Endocrinol Metab 2000; 85: 1151-1158.

39. Vicini C, et al. Transoral robotic surgery of the tongue base in obstructive sleep Apnea-Hypopnea syndrome: anatomic considerations and clinical experience. Head Neck. 2012; 34(1): 15-22.

40. Vicini $\mathrm{C}$, et al. The nose oropharynx hypopharynx and larynx (NOHL) classification: a new system of diagnostic standardized examination for OSAHS patients. Eur Arch Otorhinolaryngol. 2012; 269(4): 1297-300.

41. Weinstein GS, O’Malley Jr BW, Magnuson JS, et al. Transoral robotic surgery: a multicenter study to assess feasibility, safety, and surgical margins. Laryngoscope. 2012; 122: 1701-7.

42. Young T, Peppard PE, Gottlieb DJ. Epidemiology of obstructive sleep apnea: a population health perspective. Am J Respir Crit Care Med 2002; 165(May (9)): 1217-39.

43. Young T, Shahar E, Nieto FJ, Redline S, Newman AB, Gottlieb DJ, Walsleben JA, Finn L, Enright P, Samet JM. Predictors of sleep-disordered breathing in community-dwelling adults: the Sleep Heart Health Study. Arch Intern Med 2002; 162: 893-900.

44. Xia W, Huang Y, Peng B, Zhang X, Wu Q, Sang Y, Luo Y, Liu X, Chen Q, Tian K. Relationship between obstructive sleep apnoea syndrome and essential hypertension: a dose-response meta-analysis. Sleep Med. 2018;47:11-8. https://doi.org/10.1016/j.sleep.2018.03.016 